\title{
Thermodynamics of Acoustic Black Holes in Two Dimensions
}

\author{
Baocheng Zhang \\ School of Mathematics and Physics, China University of Geosciences, Wuhan 430074, China \\ Correspondence should be addressed to Baocheng Zhang; zhangbc.zhang@yahoo.com
}

Received 12 May 2016; Accepted 11 July 2016

Academic Editor: Emmanuel N. Saridakis

Copyright (C) 2016 Baocheng Zhang. This is an open access article distributed under the Creative Commons Attribution License, which permits unrestricted use, distribution, and reproduction in any medium, provided the original work is properly cited. The publication of this article was funded by SCOAP S $^{3}$

It is well-known that the thermal Hawking-like radiation can be emitted from the acoustic horizon, but the thermodynamic-like understanding for acoustic black holes was rarely made. In this paper, we will show that the kinematic connection can lead to the dynamic connection at the horizon between the fluid and gravitational models in two dimensions, which implies that there exists the thermodynamic-like description for acoustic black holes. Then, we discuss the first law of thermodynamics for the acoustic black hole via an intriguing connection between the gravitational-like dynamics of the acoustic horizon and thermodynamics. We obtain a universal form for the entropy of acoustic black holes, which has an interpretation similar to the entropic gravity. We also discuss the specific heat and find that the derivative of the velocity of background fluid can be regarded as a novel acoustic analogue of the two-dimensional dilaton potential, which interprets why the two-dimensional fluid dynamics can be connected to the gravitational dynamics but it is difficult for four-dimensional case. In particular, when a constraint is added for the fluid, the analogue of a Schwarzschild black hole can be realized.

\section{Introduction}

The concept of acoustic black holes, based on the kinematical analogue between the motion of sound wave in a convergent fluid flow and the motion of a scalar field in the background of Schwarzschild spacetime, had been suggested initially in 1981 [1], and then many physical systems had been used to present a similar concept, such as Bose-Einstein condensates (BEC) [2], superfluid $\mathrm{He}$ [3], slow light [4], electromagnetic waveguide [5], and light in a nonlinear liquid [6]; see review [7]. Recently, the observation of the acoustic horizon had been made in different physical systems [8-11]. But the Hawkinglike radiation $[12,13]$ is still difficult to be observed due to the small radiation temperature, while the recent report [14] on the observation of Hawking-like radiation was related to a self-amplifying mechanism called black hole laser suggested by Corley and Jacobson [15]. Thus, the analogue of black holes provides a prospective avenue to observe the Hawking radiation experimentally, since the direct observation from astrophysical black holes is nearly impossible due to the temperature that is several orders of magnitude lower than the cosmic microwave background.
As stated above, the acoustic black hole is based on only the kinematical analogue, irrelevant to the dynamics. But for Schwarzschild black holes, once the radiation starts, it will get hotter and hotter by losing energy, which is evident from the relation that the temperature is inversely proportional to the mass. This description is beyond kinematics and is mainly based on thermodynamics that is definitely dependent on the dynamics $[16,17]$. Recently, it was found that when the backreaction $[18,19]$ was considered (some different views for this were seen in [20-22], but how to discuss the back-reaction exactly is still open now [7]), the acoustic black hole will not present similar thermodynamic behaviors of Schwarzschild black holes while it looks more like a near-extremal ReissnerNordström black hole. This is an important step for the possible thermodynamics of an acoustic black hole. Next, as for thermodynamics of a gravitational black hole, the description usually has to be made with the help of proper expressions for the mass and the entropy of the acoustic black hole. Some recent developments had shown that the entropy could be endowed to an acoustic black hole by understanding the microscopical modes in some situations [23-26]. But the mass is definitely relevant to the classical dynamics, and thus 
its definition requires an analogue for gravity's equation. An interesting work [27] had been attempted for this, in which the mass and the entropy were defined by using an analogue between two-dimensional (2D) dilaton black holes and the acoustic black holes but at the same time the fluid dynamics had been fixed by the two-dimensional dilaton gravity [28]. Thus, by the analogue for gravitational dynamics, Cadoni [27] realized the thermodynamics for an acoustic black hole in two dimensions.

In the earlier discussion about thermodynamics of acoustic black holes, the dynamics is fixed in advance. In this paper, we want to study whether the dynamics of fluid can support the description of thermodynamics spontaneously only if the acoustic horizon has formed. This reminds us of a method which is well-known in gravity; that is, Einstein's equation can be derived from the thermodynamics plus the knowledge from the black hole physics [29, 30]. Thus, Einstein's equation could be regarded as a thermodynamic identity; see the review paper [31]. That means that, at the horizon, Einstein's equation is equivalent to the thermodynamic first law, and the thermodynamic quantities such as the mass and the entropy can be read off directly from Einstein's equation. Then, one might ask whether this can be extended to the fluid model, or whether it is permitted to read off the mass and the entropy of an acoustic black hole from the fluid equation. It is feasible if the kinematical analogue can give the connection between two kinds of dynamics. Moreover, the application of the method should be able to give consistent results with those obtained by other methods $[18,19,27]$. In this paper, we will try to explore these from the equation of motion for the fluid, but our discussion is limited in two dimensions. Once the mass and the entropy of the acoustic black hole are given, we can estimate the thermodynamic evolution using the specific heat, as used usually for the Schwarzschild black hole. By the estimation, we are able to find how the elements in a fluid model influence the evolution of the acoustic black hole.

The structure of the paper is as follows. We will first revisit the concept of the acoustic black hole with the model used in the initial paper of Unruh and explain the Hawking-like radiation with the perturbed action. In Section 3, we will analyze the background fluid equations and construct the kinematical connection between acoustic black holes and 2D black holes, which leads to the dynamic connection between them. Then, we identify the corresponding thermodynamic quantities by a similar method for Einstein's equation. We also discuss the same identification from the equations for BEC as an acoustic analogue of black holes. Section 4 contributes to the thermodynamic stability of the acoustic black hole with the aid of the specific heat. Finally, we discuss and summarize our results in Section 5.

\section{Acoustic Black Hole}

Consider an irrotational, barotropic fluid that was also considered in the seminal paper [1] of Unruh with the following action [19]:

$$
S=-\int d^{4} x\left[\rho \dot{\psi}+\frac{1}{2} \rho(\vec{\nabla} \psi)^{2}+u(\rho)\right],
$$

where $\rho$ is the mass density, $\psi$ is the velocity potential, that is, $\vec{v}=\vec{\nabla} \psi$, and $u(\rho)$ is the internal energy density. In this paper, we only involve the linear perturbation for the derivation of the acoustic metric, so action (1) is enough for our discussion.

The variation of $S$ will give the equations of motion, and one of them is the Bernoulli equation:

$$
\dot{\psi}+\frac{1}{2} \vec{v}^{2}+\mu(\rho)=0,
$$

where $\mu(\rho)=d u / d \rho$, and the other one is the continuity equation:

$$
\dot{\rho}+\vec{\nabla} \cdot(\rho \vec{v})=0
$$

We linearize the density and the velocity potential by replacing $\psi \rightarrow \psi+\phi$ and $\rho \rightarrow \rho+\sigma$, where $\psi$ and $\rho$ are related to the background fluid determined by the equations of motion, and $\phi$ and $\sigma$ are small perturbations. When we put the new velocity potential and mass density after perturbations into the initial action (1), a new added term, up to quadratic order, appears as

$$
S_{p}=-\frac{1}{2} \int d^{4} x\left[\rho(\vec{\nabla} \phi)^{2}-\frac{\rho}{c_{s}^{2}}(\dot{\phi}+\vec{v} \cdot \vec{\nabla} \phi)^{2}\right],
$$

where the speed of sound $c_{s}$ is defined as $c_{s}^{2}=\rho(d \mu / d \rho)$ and the equation of motion for $\phi, \phi+\left(\rho / c_{s}^{2}\right)(\dot{\phi}+\vec{v} \cdot \vec{\nabla} \phi)=0$, is used. It is noted that, for some cases such as presented in Unruh's original model [1], a completely appropriate approximation can be taken for the speed of sound as a position-independent constant, but there are also some other cases such as BEC [2, 32] which did not take the speed of sound as a constant. In this paper, we take the speed of sound as constant, in addition to the special case where the nonconstant speed of sound will be pointed out.

The propagation of sound wave on the background fields can be obtained by a wave equation, $\nabla^{2} \phi=(1 /$ $\sqrt{-g}) \partial_{\mu}\left(\sqrt{-g} g^{\mu \nu} \partial_{\nu} \phi\right)$, where $g_{\mu \nu}$ can be read off from the socalled acoustic metric:

$$
d s^{2}=\frac{\rho}{c_{s}}\left[-c_{s}^{2} d t^{2}+(d \vec{x}-\vec{v} d t)^{2}\right] .
$$

This metric, if considered as a metric of acoustic black hole, can be understood from a model called the river model of black holes $[33,34]$ : the fluid as the background is flowing along a direction to the region beyond the Newtonian escape velocity (i.e., the local velocity of sound), and the point where the velocity of background fluid equals the sound velocity represents the horizon of the black hole. From this description, it is noted that the essence of the acoustic black hole, in particular, Hawking radiation which propagates against the fluid, can be understood with a two-dimensional model. So in the paper we will consider only the case of two dimensions, for which we take the direction of fluid flowing as the $x$ axis and thus the components of the speed $\vec{v}$ along the other two directions will be suppressed naturally. Moreover, one can also reduce the dimensions by the spherical symmetry, in which this analogue will reproduce the results for the socalled "dirty black holes" [35]. 
Furthermore, if the scalar field $\phi$ is quantized, a similar effect to Hawking radiation appears at the horizon with the temperature which can be expressed as

$$
T_{a}=\left.\frac{\hbar}{4 \pi c_{s} k_{B}} \frac{d\left(c_{s}^{2}-v^{2}\right)}{d x}\right|_{v=-c_{s}}=\frac{\hbar}{2 \pi k_{B}} v^{\prime}\left(x_{h}\right),
$$

where the horizon is located at $x=x_{h}$ that is determined by $v\left(x_{h}\right)=-c_{s}$ and the prime is the derivative with regard to the coordinate $x$. A better interpretation for the origin of Hawking radiation can be from the analysis for the corrected action $S_{p}$ [22], in which some extra terms have to be added in order to ensure the positivity of perturbation modes and differentiability of the classical ground state profile (i.e., $\partial_{x} \phi$ ), and thus the superluminal modes that can overcome the frame-dragging speed and approach the horizon from the inside will be ripped apart at the horizon so that the part with positive frequencies escapes into the exterior region and the others drop again into the interior domain. This interpretation is consistent with that from the quantization, but it evidently draws support from the dispersion relation that is necessary to be considered in the realistic experiment of simulation about black/white hole horizon and that can also avoid the trans-Planckian puzzle [22].

\section{Thermodynamic Description}

At first, let us review how the Einstein equation is identified with the thermodynamic relation $[31,36,37]$. Start with the stationary metric in ADM form [38]:

$$
d s^{2}=-N_{t}(r)^{2} d t^{2}+\left[d r+N_{r}(r) d t\right]^{2}+r^{2} d \Omega^{2},
$$

where $N_{t}(r)$ and $N_{r}(r)$ are the lapse and shift functions, respectively. The metric is well behaved on the horizon, and for a four-dimensional spherical Schwarzschild solution, $N_{t}=1$ and $N_{r}=\sqrt{2 M / r}$ ( $M$ is the mass of the black hole); for a four-dimensional Reissner-Nordström solution, $N_{t}=1$ and $N_{r}=\sqrt{2 M / r-Q^{2} / r^{2}}(M$ is the mass and $Q$ is the charge of the black hole). In particular, it is noted that the acoustic metric can be obtained by taking $N_{t}=-c_{s}$ and $N_{r}=-v$ up to a conformal factor $\rho / c_{s}$.

For the metric (7), the horizon, $r=r_{h}$, is determined from the condition $N_{t}\left(r_{h}\right)-N_{r}\left(r_{h}\right)=0$. The temperature associated with this horizon is $k_{B} T_{H}=(\hbar c / 4 \pi)(d / d r)\left(N_{t}^{2}(r)-\right.$ $\left.N_{r}^{2}(r)\right)\left.\right|_{r=r_{h}}=\left(\hbar c N_{t}\left(r_{h}\right) / 2 \pi\right)\left(N_{t}^{\prime}\left(r_{h}\right)-N_{r}^{\prime}\left(r_{h}\right)\right)$, where the prime is the derivative with regard to the coordinate $r$. Consider the $(r, r)$ components of the vacuum Einstein equation, $R_{r r}=0$, and evaluate it at the horizon; this gives

$$
N_{t}\left(r_{h}\right)\left[N_{t}^{\prime}\left(r_{h}\right)-N_{r}^{\prime}\left(r_{h}\right)\right]-\frac{1}{2}=0 .
$$

Then, multiplying the equation by an imaginary displacement $d r_{h}$ of the horizon and introducing some constants, we can rewrite it as

$$
\begin{gathered}
\underbrace{\frac{\hbar c N_{t}\left(r_{h}\right)}{2 \pi}\left[N_{t}^{\prime}\left(r_{h}\right)-N_{r}^{\prime}\left(r_{h}\right)\right] \frac{c^{3}}{G \hbar} d\left(\frac{1}{4} 4 \pi r_{h}^{2}\right)}_{k_{B} T_{H}} \\
-\underbrace{\frac{1}{2} \frac{c^{4}}{G} d r_{h}}_{d S}=0
\end{gathered}
$$

and read off the expressions

$$
\begin{aligned}
& S=\frac{1}{4 l_{P}^{2}}\left(4 \pi r_{h}^{2}\right)=\frac{A_{H}}{4 l_{P}^{2}} ; \\
& E=\frac{c^{4}}{2 G} r_{h}=\frac{c^{4}}{G}\left(\frac{A_{H}}{16 \pi}\right)^{1 / 2},
\end{aligned}
$$

where $A_{H}$ is the horizon area and $l_{P}^{2}=G \hbar / c^{3}$. If the source of Einstein's equation is considered, a term $P d V$ will be added to (9) as in [36, 37], which will give an exact and strict form for the first law of thermodynamics by including the matter's influence. Here we ignore this term only for the brevity. In particular, the method is classical although we insert the Planck constant in the expressions of the temperature and the entropy by hand, which showed that the single quantity should stem from the quantum statistical mechanics. Along this line, many different situations had been discussed, which indicates the universality of this kind of identification; see review [39].

3.1. Two-Dimensional Kinematical Connection. Before applying the thermodynamic identification to acoustic black holes, we have to be sure whether there is the dynamic connection between the fluid and gravitational models only by the kinematical analogue. Now we give some direct kinematical relations between 2D black holes and acoustic black holes, which were once given in [27] but the dynamics is fixed in advance.

Generally, 4D Einstein gravity can decay into 2D by the method of spherical reduction if the line element can be written as $d s^{2}=g_{\alpha \beta} d x^{\alpha} d x^{\beta}+e^{-2 \Phi\left(x^{\alpha}\right)} d \Omega^{2}$, where $\alpha, \beta=0,1$ and $\Phi\left(x^{\alpha}\right)$ is the dilaton field. In particular, $2 \mathrm{D}$ gravity is also called dilaton gravity [28]. Start with the action considered in $[27,28]$

$$
S_{g}=\frac{1}{2} \int d^{2} x \sqrt{-g}\left(\Phi R+\lambda^{2} V(\Phi)\right)
$$

where $\Phi$ is a scalar (the dilaton), $\lambda$ is a parameter for the balance of the dimension, and $V(\Phi)$ is the dilaton potential. The equation of motion related to the variable $g_{\alpha \beta}$ is

$$
R=-\lambda^{2} \frac{d V}{d \Phi}
$$


The model admits black hole solutions with the form in Schwarzschild gauge as [40]

$$
\begin{array}{r}
d s^{2}=-\left(J(\Phi)-\frac{2 M}{\lambda}\right) d \tau^{2}+\left(J(\Phi)-\frac{2 M}{\lambda}\right)^{-1} d r^{2} \\
\Phi=\lambda r
\end{array}
$$

where $M$ is the mass of the black hole and $J=\int V d \Phi$. The location $r_{h}$ of the black hole horizon is determined by $J\left(\lambda r_{h}\right)=2 M / \lambda$. The metric is also gotten by taking $N_{t}=1$ and $N_{r}=\sqrt{1-J-2 M / \lambda}$ from the $\operatorname{ADM}$ form (7), but its relation to the $2 \mathrm{D}$ section of Schwarzschild black hole is subtle, which will be involved later.

Comparing the metric (13) with the acoustic metric (5) in two dimensions, the kinematical relation can be gotten as

$$
\begin{aligned}
& r \sim \int \rho d x, \\
& \tau \sim t+\int d x \frac{v}{c_{s}^{2}-v^{2}}, \\
& J \sim \frac{2 M}{\lambda}+\frac{\rho}{c_{s}}\left(c_{s}^{2}-v^{2}\right),
\end{aligned}
$$

as presented in [27].

When we endow the thermodynamic-like description to the acoustic black hole, the role played by relations (14) will be seen. Now we turn to another kinematical relation. As stated in $[16,17]$, Hawking radiation is purely kinematic effect, and so is the temperature. Thus, one can relate the temperature $T_{b}=(\lambda / 4 \pi) V\left(\lambda r_{h}\right)$ for the $2 \mathrm{D}$ black holes $[41,42]$ with that in (6) for the acoustic black hole by

$$
\frac{\hbar}{2 \pi k_{B}} v^{\prime}\left(x_{h}\right) \sim \frac{\lambda}{4 \pi} V\left(\lambda r_{h}\right),
$$

which, together with the fluid's equation at the horizon where the two equations of motion for the fluid have the same form, gives a constraint for the fluid dynamics at the horizon. But it has to be stressed that this is not evident for 4D situation, since the conformal factor (i.e., irrelevant to the kinematical results but can influence the dynamics) is not treated properly in kinematical analogue there. Therefore, one has to be careful about 4D situation, but at least in two dimensions, we can proceed to the discussion about thermodynamics for acoustic black holes.

3.2. Correspondence between Full Actions. As well known, the acoustic metric is obtained through the linear perturbation, so in this subsection we will give a brief proof that, at the perturbative level, the two cases are still equivalent. For the acoustic fluid, the corrected action is given by (4), and in two dimensions, the expression is

$$
S_{P}=-\frac{1}{2} \int d^{2} x A\left[\rho\left(\partial_{x} \phi\right)^{2}-\frac{\rho}{c_{s}^{2}}\left(\partial_{t} \phi+v \cdot \partial_{x} \phi\right)^{2}\right],
$$

where $A$ is the cross-sectional area of fluid and $\phi$ is the perturbative field whose equation of motion is given by

$$
\nabla^{2} \phi=\frac{1}{\sqrt{-g}} \partial_{\mu}\left(\sqrt{-g} g^{\mu \nu} \partial_{\nu} \phi\right)=0 .
$$

For 2D dilaton gravity, the corrected action can be given by the Polyakov-Liouville action (see [28] and the references therein):

$$
I_{\mathrm{PL}}=-\frac{\alpha}{2 \pi} \int d^{2} x \sqrt{-g}\left(\frac{(\nabla \varphi)^{2}}{2}+\varphi R\right),
$$

where $\alpha$ is quantum coupling parameter related to the number of conformal scalar fields, $R$ is $2 \mathrm{D}$ scalar curvature, and $\varphi$ field is related to the back-reaction of spacetime caused by Hawking radiation. The equation of motion for the $\varphi$ field is

$$
\nabla^{2} \varphi=R
$$

Within the Schwarzschild gauge,

$$
d s^{2}=-X(r) d \tau^{2}+\frac{1}{X(r)} d r^{2}
$$

(17) becomes

$$
-\frac{1}{X} \partial_{\tau}^{2} \phi+\frac{d X}{d r} \frac{d \phi}{d r}+X \partial_{r}^{2} \phi=0
$$

and (19) becomes

$$
-\frac{1}{X} \partial_{\tau}^{2} \varphi+\frac{d X}{d r} \frac{d \varphi}{d r}+X \partial_{r}^{2} \varphi=R=-\frac{d^{2} X}{d r^{2}} .
$$

Then, we can relate the two equations with $\phi=\varphi+$ $\ln X$. Since $X$ is independent of the time, the two fields $\phi$ and $\varphi$ have the same behaviors at the thermodynamic or dynamic level. Furthermore, we can see the equivalence by the respective numbers of degree of freedom; that is, for the fluid model, there are three parameters-the fluid speed $v$, the fluid density $\rho$, and the perturbative field $\phi$; for $2 \mathrm{D}$ dilaton gravity, there are also three parameters-the dilaton field $\Phi$, the metric field $g_{\mu \nu}$ (only one component in the Schwarzschild gauge), and the Polyakov-Liouville field $\varphi$. But for $4 \mathrm{D}$ situation, there is never so nice correspondence, and even for the 4D Schwarzschild black holes, the gravitational parameters can not be modeled by the fluid parameters directly, which requires conformal parameters to relate them, but the conformal parameter is dependent on the time, so one must be careful to treat $4 \mathrm{D}$ situation, in particular at the level of thermodynamics.

3.3. Thermodynamic Identification. As far as we know, the method of thermodynamic identification has not been applied for 2D gravity. Here we give a brief implementation for this. At the horizon of black hole (13), the field equation for 2D dilaton gravity model (11) becomes

$$
\left.\frac{d J}{d r}\right|_{r_{h}}=\lambda V\left(\lambda r_{h}\right)
$$


When the imaginary displacement $d r_{h}$ of the horizon is included, the equation is reexpressed with the form

$$
\underbrace{\frac{\hbar \lambda}{4 \pi} V\left(\lambda r_{h}\right) \frac{2 \pi \lambda}{\hbar} d r_{h}}_{k_{B} T_{b}}-\underbrace{\frac{\lambda}{2} d J\left(\lambda r_{h}\right)}_{d S_{b}}=0 .
$$

From this, one can read off

$$
\begin{aligned}
& S_{b}=\frac{2 \pi \lambda}{\hbar} r_{h}, \\
& E_{b}=\frac{\lambda}{2} J\left(\lambda r_{h}\right),
\end{aligned}
$$

up to an integral constant, respectively. In particular, they are consistent with the expressions of entropy and energy obtained by using other methods [41, 42].

Now we begin to discuss the thermodynamics for an acoustic black hole. Taking the derivative with regard to $x$ for the steady Bernoulli equation (2), we have Euler's equation in two dimensions:

$$
\rho v \frac{d v}{d x}+c_{s}^{2} \frac{d \rho}{d x}=0
$$

which is one of the equations of motion in two dimensions but it is consistent with the other one at the horizon. Considering the expression of the temperature (6), we can rewrite the equation at the horizon as

$$
\underbrace{\frac{\hbar}{2 \pi} v^{\prime}\left(x_{h}\right)}_{k_{B} T_{a}} \underbrace{\frac{2 \pi}{\hbar K} \rho\left(x_{h}\right) d x_{h}}_{d S_{a}}-\underbrace{\frac{c_{s}}{K} d \rho\left(x_{h}\right)}_{d E_{a}}=0,
$$

where $K$ is introduced in order to balance the dimension. Then, we read off

$$
\begin{aligned}
& S_{a}=\frac{2 \pi}{\hbar K} \int^{x_{h}} \rho(x) d x ; \\
& E_{a}=\frac{c_{s}}{K} \int^{x_{h}} \rho^{\prime}(x) d x,
\end{aligned}
$$

where the integral is made near the horizon. Then, we attempt to understand these identifications (28). The mass can be interpreted with a force-density term in Euler's equation [7]; that is, $c_{s}^{2}(d \rho / d x)=\delta E_{a} / \delta x=F_{x} \equiv-\rho(d \mu / d \rho)(d \rho / d x)$. Here it is also regarded as the acoustic analogue of gravitational mass of black holes. The entropy can be interpreted as from the property of the horizon, but it can also be interpreted with other ways, that is, brick wall model [23-26] that assumes the black hole is under an equilibrium state with the thermal gas surrounding it. Note that our identification for the entropy of the acoustic black hole is formally different from that from brick wall model $[24,25]$, but one can obtain a consistent form from the two methods when the system's evolution is considered; that is, $\dot{S}_{a} \propto \kappa$ (i.e., the surface gravity of acoustic black holes via $T=\kappa / 2 \pi$ ) by using the results of [19] for back-reaction expression of $\rho$ or $v$, in line with $[23,24]$.
It is evident that the thermodynamics of acoustic black holes cannot be Schwarzschild-like, since the Schwarzschild analogue usually takes $\rho(x) \propto x^{-3 / 2}$ and $v(x) \propto x^{-1 / 2}$ up to a conformal difference. One might suspect that this inconsistency is due to the one-dimensional linear fluid model that we take approximately (see the discussion below metric (5)), but the same mathematical forms will also be gotten by reducing action (1) with the consideration of spherical symmetry, since $\vec{\nabla} \psi=(\partial \psi / \partial x) \hat{x}+(\partial \psi / \partial y) \hat{y}+(\partial \psi / \partial z) \widehat{z}=(\partial \psi / \partial r) \widehat{r}+(1 /$ $r)(\partial \psi / \partial \theta) \hat{\theta}+(1 / r \sin \theta)(\partial \psi / \partial \phi) \widehat{\phi}$ which reduces to $\vec{\nabla} \psi=$ $(\partial \psi / \partial x) \hat{x}$ for the case discussed in the paper or $\vec{\nabla} \psi=(\partial \psi /$ $\partial r) \widehat{r}$ when the velocity is suppressed in two angular directions. Thus, if a two-dimensional fluid model is considered, it might not be related to the simple reduction of fourdimensional gravity, described by (8), so its thermodynamics does not have to be Schwarzschild-like.

As a consistent check, we will see that the kinematical relation (14) can give the thermodynamic connection between the 2D dilaton black hole and the acoustic black hole; that is, taking $\lambda \sim 1 / K$, the relation $r \sim \int \rho d x$ indicates $S_{b} \sim S_{a}$; from the relation $J \sim 2 M / \lambda+\left(\rho / c_{s}\right)\left(c_{s}^{2}-v^{2}\right)$, one has $d J \sim 2 \rho d v \sim 2 c_{s} d \rho$ at the horizon, which indicates $E_{b} \sim E_{a}$. Therefore, the kinematical relations lead to a direct connection between the thermodynamics of $2 \mathrm{D}$ dilaton black hole and acoustic black hole, under the condition that any relation between fluid dynamics and gravitational dynamics is not assumed in advance.

In order to present the universal property of thermodynamic identification (28), we will also include the external potential explicitly in the discussion of acoustic black hole with another kind of popular model that is related to BEC. For the analogue of BEC, the corresponding Euler equation can be expressed as $[2,32]$

$$
\rho_{B} v_{B} \frac{d v_{B}}{d x}+\frac{\rho_{B}}{m} \frac{d V_{\mathrm{ext}}}{d x}+c_{B}^{2} \frac{d \rho_{B}}{d x}=0
$$

This can be obtained from the Gross-Pitaevskii equation, $i \hbar \partial_{t} \Psi=\left(-\left(\hbar^{2} / 2 m\right) \nabla^{2}+V_{\text {ext }}+\left(4 \pi a \hbar^{2} / m\right)|\Psi|^{2}\right) \Psi$, where the condensate is considered in the dilute gas approximation and nearly all atoms are in the same single-particle quantum state $\Psi(x, t), m$ is the mass of individual atoms, $a$ is the scattering length, and $V_{\text {ext }}(x)$ is the external potential that trapped these bosons. In particular, if a background stationary state, $\Psi_{B}(x, t)=\sqrt{\rho_{B}(x)} e^{i \psi_{B}(x)} e^{-i \omega t}$, is considered, the propagation of small perturbations of the condensate around this background can be calculated to get the acoustic metric, as presented in (5). The velocity of the background is given by $v_{B}=(\hbar / m) \nabla \psi_{B}(x)$ and the local velocity of sound by $c_{B}(x)=$ $(\hbar / m) \sqrt{4 \pi a \rho_{B}(x)}$. Moreover, the quantum pressure term $Q(\vec{x})=-\left(\hbar^{2} / 2 m\right)\left(\nabla^{2} \sqrt{\rho_{B}(\vec{x})} / \sqrt{\rho_{B}(\vec{x})}\right)$ is ignored since BEC for the analogue of acoustic black holes always works within the regime of validity of Thomas-Fermi approximation [43] that the condensate does not vary on length scales shorter than the healing length $\xi=1 / \sqrt{8 \pi \rho_{B} a}$. 
With the temperature of the acoustic black hole known in advance (note that the speed of sound here is not constant), (29) can be rewritten at the horizon as

$$
\begin{aligned}
\underbrace{\frac{\hbar}{2 \pi}\left(v_{B}^{\prime}\left(x_{h}\right)+c_{B}^{\prime}\left(x_{h}\right)\right)}_{k_{B} T} \underbrace{\frac{2 \pi}{\hbar K} \rho_{B}\left(x_{h}\right) d x}_{d S_{B}} \\
-\underbrace{\left(\frac{\rho_{B}\left(x_{h}\right)}{K m c_{B}\left(x_{h}\right)} d V_{\mathrm{ext}}\left(x_{h}\right)+\frac{3 c_{B}\left(x_{h}\right)}{2 K} d \rho_{B}\left(x_{h}\right)\right)}_{d E_{B}}
\end{aligned}
$$$$
=0 .
$$

It is easily seen that the identification of the entropy is the same with that in (28). The mass is expressed as

$$
\begin{aligned}
& E_{B} \\
& =\frac{1}{K} \int^{x_{h}}\left[\frac{\rho_{B}\left(x_{h}\right)}{m c_{B}\left(x_{h}\right)} d V_{\text {ext }}\left(x_{h}\right)+\frac{3 c_{B}\left(x_{h}\right)}{2} d \rho_{B}\left(x_{h}\right)\right],
\end{aligned}
$$

where the expression of $E_{B}$ is different from that of $E_{a}$ due to the nonconstant speed of sound and the external potential. But it is noted that the expression of entropy is modelindependent. This can also be understood by the entropic gravity [44], which assumed that the gravity is derived from the change of entropy with the form $\Delta S=2 \pi k_{B}(\Delta x / \lambda)$. Thus, if we take the proper form for the constant $K$ and the relation $r \sim \int \rho d x$, the entropy in (28) is consistent with $\Delta S$, which shows again that the thermodynamic-like description for analogous black hole is feasible at least in 2D.

\section{Specific Heat}

From the discussion above, we have known the expressions of thermodynamic quantities of acoustic black holes which conform to the first law of thermodynamics. Actually, more importantly, we want to discuss how to use these quantities to describe the evolution of acoustic physical systems, that is, the change caused by emission of thermal radiation from the acoustic horizon. The calculation of back-reaction provided a fundamental method to answer this question, but here we want to estimate it via specific heat of an acoustic black hole, which will give the information about the change of temperature during the radiation. Generally, the temperature in black hole theory is a geometric quantity related closely to the spacetime background, so the change of temperature will indicate the change of spacetime background that is also called back-reaction if the change is not so violent.

According to our results in (6) and (28), the specific heat of an acoustic black hole can be written as

$$
C_{a}=\frac{d E_{a}}{d T_{a}}=\frac{d E_{a} / d x}{d T_{a} / d x}=-\frac{2 \pi k_{B} \rho\left(x_{h}\right)}{\hbar K} \frac{v^{\prime}\left(x_{h}\right)}{v^{\prime \prime}\left(x_{h}\right)} .
$$

It is easily seen that the sign of specific heat is dependent on the first and second derivatives of the velocity with regard to the coordinate $x$. If we take the model of Laval nozzle that is described in [19], which gave $v^{\prime}\left(x_{h}\right)>0$ and $v^{\prime \prime}\left(x_{h}\right)<0$, we find a positive specific heat. This means that the temperature will decrease after the radiation is emitted, and such behavior of acoustic black holes resembles a near-extremal ReissnerNordström black hole but not a Schwarzschild black hole, which is consistent with the result of back-reaction analysis [19]. Thus, it indicates that the thermodynamics of acoustic black holes is model-dependent. The extension to the case where the speed of sound is not constant is possible and will not change our conclusion about the model-dependent thermodynamic-like behaviors for the corresponding acoustic black holes.

The specific heat of $2 \mathrm{D}$ dilaton black holes is also easy to be gotten:

$$
C_{b}=\frac{d E_{b}}{d T_{b}}=2 \pi \frac{V\left(\Phi_{h}\right)}{d V\left(\Phi_{h}\right) / d \Phi}
$$

which is consistent with that obtained in $[45,46]$. In particular, the specific heat is dependent on the dilaton potential which can lead to a model similar to the near-extremal Reissner-Nordström black hole under some conditions. Then, whether the two specific heats have any relation under the kinematical analogue, a straight use of kinematical relations in (15) and $r \sim \int \rho d x$ shows $C_{a} \sim C_{b}$, which is further evidence for the connection between the two dynamics with only the kinematical analogue made in advance.

From the analysis above, it is seen that the derivative of the parameter $v$ in the fluid can be regarded as the analogue of dilaton potential, which is equivalent to such analogue, $v \sim M$, where $M$ is the mass of $2 \mathrm{D}$ dilaton black hole. This is different from the usual kinematical analogue for $2 \mathrm{D}$ sections of Schwarzschild black hole; that is, $v \sim \sqrt{2 M_{H} / r_{H}}$, where $M_{H}$ and $r_{H}$ are the mass and the radial coordinate of Schwarzschild black hole, respectively. In fact, a specific situation for $2 \mathrm{D}$ dilaton black hole, that is, $V(\Phi)=1 / \sqrt{2 \Phi}$, can be taken in the Schwarzschild form by making the transformations $g_{\mu \nu} \rightarrow(1 / \sqrt{2 \Phi}) g_{\mu \nu}$ and $r \rightarrow(\lambda / 2) r_{H}^{2}$ for the metric (13). This means the kinematical relation in (15) becomes $\left(\hbar / 2 \pi k_{B}\right) v^{\prime}\left(x_{h}\right) \sim(\lambda / 4 \pi)\left(1 / \sqrt{2 \Phi_{h}}\right) \sim\left(\hbar / 8 \pi G k_{B} M\right)$, where the speed of light is taken as $c=1$. As discussed in the last section, the kinematical relations can lead to the relation between the mass of acoustic black hole and the gravitational black hole, so we have $v^{\prime}\left(x_{h}\right) \sim 1 / 4 G E_{a} \sim 1$ / $-4 \int^{x_{h}} \rho(x) v^{\prime}(x) d x$. Thus, the relation for the parameter $v$ in the fluid is

$$
-4 v^{\prime}\left(x_{h}\right) \int^{x_{h}} \rho(x) v^{\prime}(x) d x \sim 1,
$$

which is necessary to model the thermal behavior of a Schwarzschild black hole. With this relation, it is easy to confirm that $C_{a}<0$. Of course, one can also obtain a similar relation for the other physical systems like BEC to attempt to find the Hawking-like radiation as emitted from a Schwarzschild black hole. Further, as seen from the transformation for 2D Schwarzschild metric into dilaton black hole metric, a conformal factor related to the dilaton potential is involved. Thus, it is understood why the acoustic black holes always present directly the behaviors of 2D dilaton black holes, 
while an extra constraint is required for the simulation of Schwarzschild-like black holes.

\section{Conclusion}

In this paper, we have reinvestigated the concept of acoustic black holes and discussed background fluid equations. Even though the fluid equations cannot give the complete expressions for each field without any extra knowledge besides the equations of motion, they still include a wealth of information. Via fluid's equation of motion as a thermodynamic identity, we have read off the mass and the entropy for acoustic black holes with the temperature known in advance. This identification is guaranteed by the kinematical connection which can lead to the dynamic connection between the fluid and gravitational models in two dimensions. In particular, through the analysis for the kinematical relations, we have found that the thermodynamics of acoustic black holes reproduces the thermodynamics of two-dimensional dilaton black holes exactly, and so the fluid can be regarded as a natural analogue of two-dimensional dilaton gravity, which is significant for many ongoing related experimental observations. Novelly, we have also found that the entropy for acoustic black holes is model-independent and has an interpretation similar to the entropic gravity. Moreover, it is found that the derivative of velocity $v$ of the background fluid is a nice analogue of the dilaton potential, from the kinematical and thermodynamic perspective, respectively, which also interprets why it is difficult for the $4 \mathrm{D}$ kinematical analogue to lead to any dynamic analogue, due to conformal difference.

With the mass and the temperature identified by thermodynamics, we have proceeded to get the specific heat for the acoustic black hole and found the sign of the specific heat is model-dependent, which means that some extra knowledge besides the equations of motion must be given to estimate the thermodynamic stability. However, when we want to model some kind of black holes, that is, Schwarzschild black holes, the relation about the parameters in the fluid can be known only by the fluid equation and the thermodynamic correspondence, as made in (34). Finally, all our analysis is made for $2 \mathrm{D}$ model, so whether they can be extended to $4 \mathrm{D}$ or higher dimensions is unclear now. It might be possible, however, to relate $2 \mathrm{D}$ with $4 \mathrm{D}$ cases by taking into account properly the behavior of $2 \mathrm{D}$ dilaton gravity under Weyl rescaling of the metric [45], which deserves a further investigation.

\section{Competing Interests}

The author declares that they have no competing interests.

\section{Acknowledgments}

The author acknowledges the support by Grant no. 11374330 from the National Natural Science Foundation of China and from Open Research Fund Program of the State Key Laboratory of Low-Dimensional Quantum Physics and from the
Fundamental Research Funds for the Central Universities, China University of Geosciences (Wuhan) (no. CUG150630).

\section{References}

[1] W. G. Unruh, "Experimental black-hole evaporation?" Physical Review Letters, vol. 46, no. 21, pp. 1351-1353, 1981.

[2] L. J. Garay, J. R. Anglin, J. I. Cirac, and P. Zoller, "Sonic analog of gravitational black holes in Bose-Einstein condensates," Physical Review Letters, vol. 85, no. 22, pp. 4643-4647, 2000.

[3] T. A. Jacobson and G. E. Volovik, "Event horizons and ergoregions in $\mathrm{He}^{3}$," Physical Review D, vol. 58, Article ID 064021, 1998.

[4] W. G. Unruh and R. Schützhold, "On slow light as a black hole analogue," Physical Review D, vol. 68, no. 2, Article ID 024008, 14 pages, 2003.

[5] R. Schützhold and W. G. Unruh, "Hawking radiation in an electromagnetic waveguide?" Physical Review Letters, vol. 95, Article ID 031301, 2005.

[6] M. Elazar, V. Fleurov, and S. Bar-Ad, "All-optical event horizon in an optical analog of a Laval nozzle," Physical Review A, vol. 86, no. 6, Article ID 063821, 5 pages, 2012.

[7] C. Barceló, S. Liberati, and M. Visser, "Analogue gravity," Living Reviews in Relativity, vol. 14, article 3, 2011.

[8] T. G. Philbin, C. Kuklewicz, S. Robertson, S. Hill, F. König, and U. Leonhardt, "Fiber-optical analog of the event horizon," Science, vol. 319, no. 5868, pp. 1367-1370, 2008.

[9] O. Lahav, A. Itah, A. Blumkin et al., "Realization of a sonic black hole analog in a Bose-Einstein condensate," Physical Review Letters, vol. 105, no. 24, Article ID 240401, 4 pages, 2010.

[10] F. Belgiorno, S. L. Cacciatori, M. Clerici et al., "Hawking radiation from ultrashort laser pulse filaments," Physical Review Letters, vol. 105, no. 20, Article ID 203901, 2010.

[11] B. Horstmann, B. Reznik, S. Fagnocchi, and J. I. Cirac, "Hawking radiation from an acoustic black hole on an ion ring," Physical Review Letters, vol. 104, no. 25, Article ID 250403, 2010.

[12] S. W. Hawking, "Black hole explosions?" Nature, vol. 248, no. 5443, pp. 30-31, 1974

[13] S. W. Hawking, "Particle creation by black holes," Communications in Mathematical Physics, vol. 43, no. 3, pp. 199-220, 1975.

[14] J. Steinhauer, "Observation of self-amplifying Hawking radiation in an analogue black-hole laser," Nature Physics, vol. 10, no. 11, pp. 864-869, 2014.

[15] S. Corley and T. Jacobson, "Black hole lasers," Physical Review D, vol. 59, no. 12, Article ID 124011, 1999.

[16] M. Visser, "Acoustic black holes: horizons, ergospheres and Hawking radiation," Classical and Quantum Gravity, vol. 15, no. 6, pp. 1767-1791, 1998.

[17] M. Visser, "Hawking radiation without black hole entropy," Physical Review Letters, vol. 80, no. 16, pp. 3436-3439, 1998.

[18] R. Balbinot, S. Fagnocchi, A. Fabbri, and G. P. Procopio, "Backreaction in acoustic black holes," Physical Review Letters, vol. 94, no. 16, Article ID 161302, 4 pages, 2005.

[19] R. Balbinot, S. Fagnocchi, and A. Fabbri, "Quantum effects in acoustic black holes: the backreaction," Physical Review D, vol. 71, no. 6, Article ID 064019, 2005.

[20] M. Stone, "Acoustic energy and momentum in a moving medium," Physical Review E, vol. 62, no. 1, pp. 1341-1350, 2000.

[21] R. Schützhold, M. Uhlmann, Y. Xu, and U. R. Fischer, "Quantum backreaction in dilute Bose-Einstein condensates," Physical Review D, vol. 72, no. 10, Article ID 105005, 8 pages, 2005. 
[22] C. Maia and R. Schützhold, "Quantum toy model for blackhole backreaction," Physical Review D, vol. 76, no. 10, Article ID 101502, 2007.

[23] S. Giovanazzi, "Entanglement entropy and mutual information production rates in acoustic black holes," Physical Review Letters, vol. 106, no. 1, Article ID 011302, 2011.

[24] M. Rinaldi, "Entropy of an acoustic black hole in Bose-Einstein condensates," Physical Review D, vol. 84, no. 12, Article ID 124009, 5 pages, 2011.

[25] M. Rinaldi, "The entropy of an acoustic black hole in boseeinstein condensates: transverse modes as a cure for divergences," International Journal of Modern Physics D, vol. 22, no. 4, Article ID 1350016, 12 pages, 2013.

[26] M. A. Anacleto, F. A. Brito, E. Passos, and W. P. Santos, "The entropy of the noncommutative acoustic black hole based on generalized uncertainty principle," Physics Letters B, vol. 737, pp. 6-11, 2014.

[27] M. Cadoni, "Acoustic analogues of two-dimensional black holes," Classical and Quantum Gravity, vol. 22, no. 2, pp. 409419, 2005.

[28] D. Grumiller, W. Kummer, and D. V. Vassilevich, "Dilaton gravity in two dimensions," Physics Reports, vol. 369, no. 4, pp. 327430, 2002.

[29] T. Jacobson, "Thermodynamics of spacetime: the Einstein equation of state," Physical Review Letters, vol. 75, no. 7, pp. 12601263, 1995.

[30] C. Eling, R. Guedens, and T. Jacobson, "Nonequilibrium thermodynamics of spacetime," Physical Review Letters, vol. 96, no. 12, Article ID 121301, 4 pages, 2006.

[31] T. Padmanabhan, "Gravity and the thermodynamics of horizons," Physics Reports, vol. 406, no. 2, pp. 49-125, 2005.

[32] C. Barceló, S. Liberati, and M. Visser, "Analogue gravity from Bose-Einstein condensates," Classical and Quantum Gravity, vol. 18, no. 6, pp. 1137-1156, 2001.

[33] A. J. S. Hamilton and J. P. Lisle, "The river model of black holes," American Journal of Physics, vol. 76, article 519, 2008.

[34] S. del Campo, R. Herrera, S. Lepe, and J. Saavedra, "Role of the Gullstrand-Painlevè metric in acoustic black holes," Journal of Physics: Conference Series, vol. 134, no. 1, Article ID 012006, 2008.

[35] M. Visser, "Dirty black holes: thermodynamics and horizon structure," Physical Review D, vol. 46, no. 6, pp. 2445-2451, 1992.

[36] T. Padmanabhan, "Classical and quantum thermodynamics of horizons in spherically symmetric spacetimes," Classical and Quantum Gravity, vol. 19, no. 21, pp. 5387-5408, 2002.

[37] D. Kothawala, S. Sarkar, and T. Padmanabhan, "Einstein's equations as a thermodynamic identity: the cases of stationary axisymmetric horizons and evolving spherically symmetric horizons," Physics Letters B, vol. 652, no. 5-6, pp. 338-342, 2007.

[38] P. Kraus and F. Wilczek, "Self-interaction correction to black hole radiance," Nuclear Physics B, vol. 433, no. 2, pp. 403-420, 1995.

[39] T. Padmanabhan, “Thermodynamical aspects of gravity: new insights," Reports on Progress in Physics, vol. 73, no. 4, Article ID 046901, 2010.

[40] D. Louis-Martinez and G. Kunstatter, "Birkhoff's theorem in two-dimensional dilaton gravity," Physical Review D, vol. 49, no. 10, pp. 5227-5230, 1994.

[41] R. B. Mann, "Conservation laws and two-dimensional black holes in dilaton gravity," Physical Review D, vol. 47, no. 10, pp. 4438-4442, 1993.
[42] R. C. Myers, "Black hole entropy in two dimensions," Physical Review D, vol. 50, no. 10, pp. 6412-6421, 1994.

[43] F. Dalfovo, S. Giorgini, L. P. Pitaevskii, and S. Stringari, "Theory of Bose-Einstein condensation in trapped gases," Reviews of Modern Physics, vol. 71, no. 3, pp. 463-512, 1999.

[44] E. Verlinde, "On the origin of gravity and the laws of Newton," Journal of High Energy Physics, vol. 2011, article 29, 2011.

[45] M. Cadoni, "Conformal equivalence of 2D dilaton gravity models," Physics Letters B, vol. 395, no. 1-2, pp. 10-15, 1997.

[46] Y. S. Myung, Y.-W. Kim, and Y.-J. Park, "Thermodynamic duality between RN black hole and 2D dilaton gravity," Modern Physics Letters A, vol. 23, no. 2, pp. 91-98, 2008. 

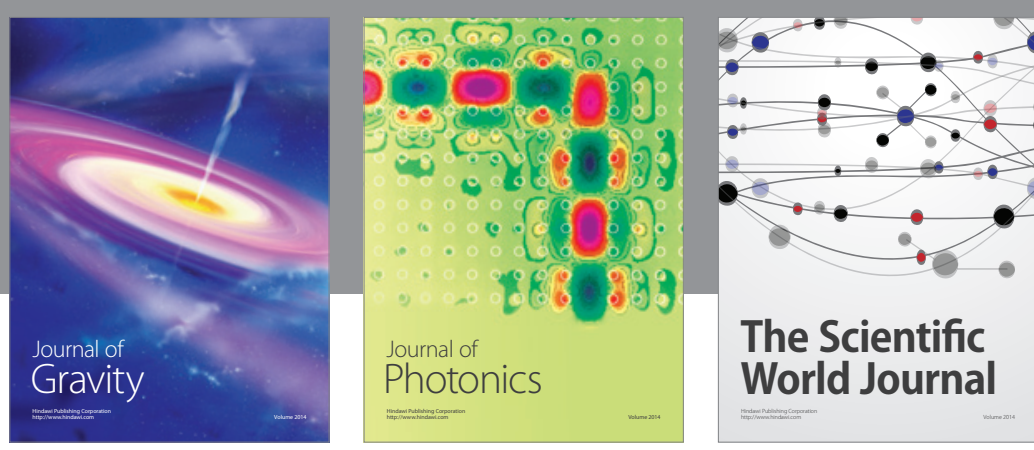

The Scientific World Journal
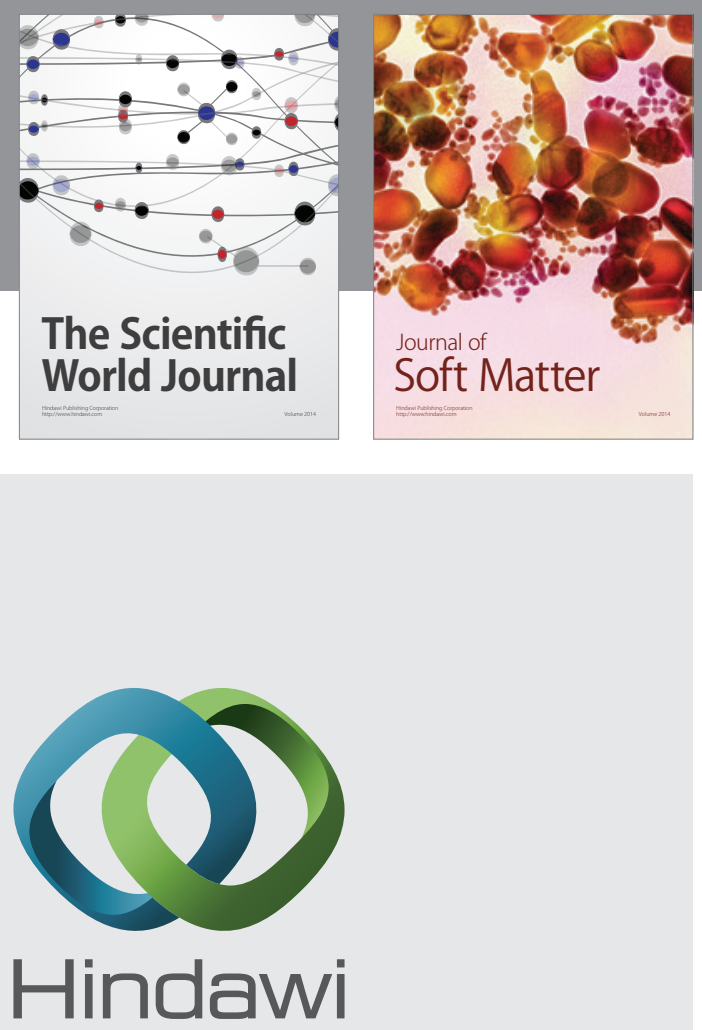

Submit your manuscripts at

http://www.hindawi.com

nternational Journal of

Statistical Mechanics
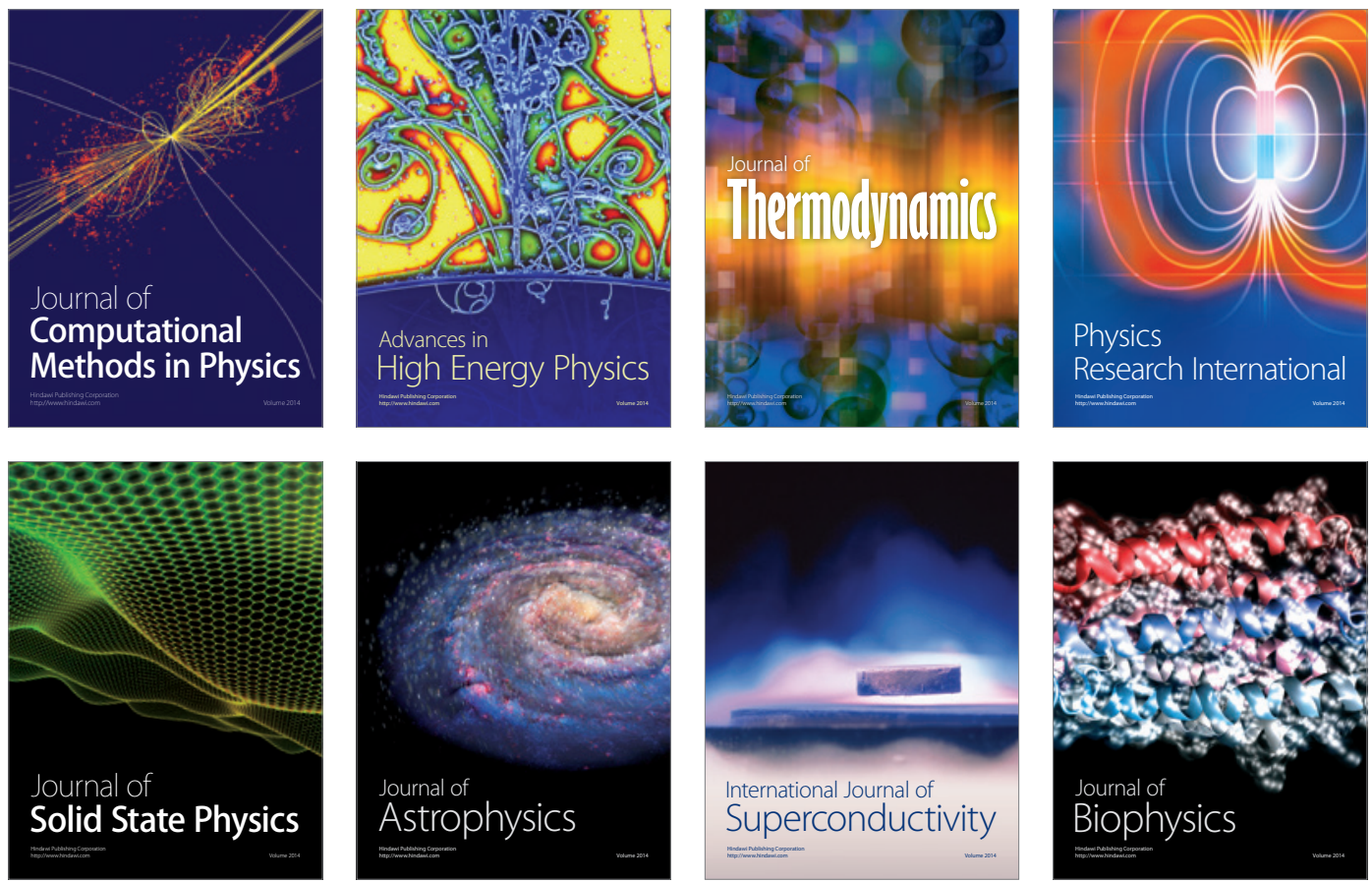
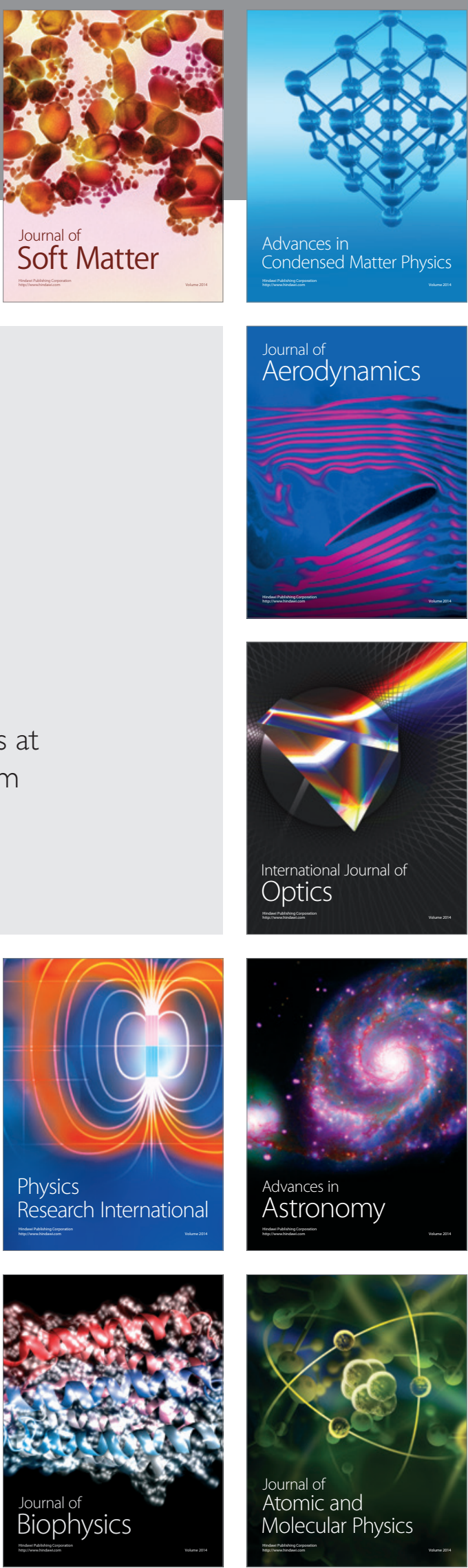